\title{
Quality Improvement in the Neonatal ICU
}

In this issue of Respiratory CARe Powell et al ${ }^{1}$ report a reduction in unplanned extubations in the neonatal ICU using lean methodology. This study is important on a number of levels. It is local research requiring fewer resources by using the lean methodology and applying a rapid cycle of plan, do, study, and act (PDSA). This method, ${ }^{2}$ also known as the Deming cycle for J. Edward Deming, was a modification of the Shewhart cycle. These quality improvement processes were initially implemented on a large scale in Japan, which resulted in a significant improvement in the quality of automobile manufacturing. Subsequently, industries around the world adopted quality improvement cycles, resulting in an improvement of performance in many industries, including health care. The idea is that small-scale tests are studied and fine-tuned before being followed by more small-scale tests. An iterative approach ${ }^{2}$ is used to optimize a range of changes until confidence is developed that widespread implementation will produce the greatest improvement possible. Importantly, in this study, PDSA cycles were useful for changing site-specific practices to reduce the serious and potentially life-threatening consequences ${ }^{3,4}$ associated with unplanned extubation. As clinicians, we can participate in research like this, in a quality improvement process that will increase our success in saving lives. The success reported was a result of local actions taken in association with local research that clinicians performed in their own facility.

It is not clear from the report whether an iterative PDSA process that involved pretesting cycles and optimization was actually used before the wider implementation. A previously reported case study ${ }^{3}$ that did report 2 cycles of tube-securing interventions resulted in a reduction of unplanned extubations from 4.8 to 3.4 with the first intervention and on down to 0.9 after a second intervention. Nevertheless, consider what this study demonstrated: a reduction in unplanned extubations and a statistically significant decrease in intubation days, confirming the successes reported by others ${ }^{3,5}$ using similar PDSA processes. The authors documented and evaluated all of the intubated infants in their facility from November 1, 2012, to June 6, 2014.

The authors have disclosed no conflicts of interest.

Correspondence: James L Hulse PhD MPH RRT-NPS RPFT, Oregon Institute of Technology, 212E Dow Center for Health Professions, 3201 Campus Dr., Klamath Falls, OR 97601-8801. E-mail: james.hulse@oit.edu.

DOI: $10.4187 /$ respcare. 05285
They found a baseline rate of 3.8 unplanned extubations per 100 intubation days. Then they identified several possible causes, such as small endotracheal tubes (ETTs), limited skin surface for securing ETTs, high humidity in the incubator, high frequency of repositioning, infant growing, and so on. Following team conferences, the staff identified these areas for improvement: standardized protocol for turning intubated infants by 2 caregivers, weekly assessment of ETT placement with growth, communication between registered nurse and respiratory therapist regarding tube positon, a respiratory therapist-driven weaning protocol, ETT migration investigation, a standardized taping method, and a requirement for respiratory therapists to hold the infant head position stable for $\mathrm{x}$-ray on all intubated patients.

See the Original Study on Page 1567

Subsequently, the 2-month data for post-intervention was collected and compared with the pre-intervention phase. The unplanned extubation numbers were reduced significantly from 3.8 to 2.7 unplanned extubations/100 intubation days pre- and post-intervention, respectively. Although the post-intervention data collection was limited, these findings seem promising. The authors have indicated that staff continue to identify interventions, which suggests that the iterations are continuing. We are curious whether the authors could do a follow-up evaluation and provide the long-term effects of their initial and ongoing interventions. Another interesting finding that is worth considering is that $26 \%$ of the unplanned extubation cases did not need re-intubation. Rates reported for re-intubation ${ }^{6}$ of planned extubations are $<74 \%$, which suggests that the unplanned extubations were indeed premature.

Large prospective randomized studies with controls are essential in establishing our practice. In addition, clinicians cannot provide the best care unless they also study their own practices and fine tune them. We should reflect on this article by considering what magnitude of improvements might develop if more hospitals and clinicians used the PDSA process. This study adds to a growing number of studies demonstrating the success of individual neonatal ICUs in reducing unplanned extubations by applying quality improvement processes.

James L Hulse PhD MPH RRT-NPS RPFT Oregon Institute of Technology Klamath Falls, Oregon 


\section{EDITORIALS}

Nam Hai Mai MD PhD

Oregon Institute of Technology

Klamath Falls, Oregon

\section{REFERENCES}

1. Powell B, Gilbert E, Vosko TA. Reducing unplanned extubations in the NICU using lean methodology. Respir Care 2016;61(12): $\bullet \bullet$.

2. Taylor MJ, McNicholas C, Nicolay C, Darzi A, Bell D, Reed JE. Systematic review of the application of the plan-do-study-act method to improve quality in healthcare. BMJ Qual Saf 2014;23(4):290-298.
3. Loughead JL, Brennan RA, DeJuilio P, Camposeo V, Wengert J, Cooke D. Reducing accidental extubations in neonates. Jt Comm J Qual Patient Saf 2008;34(3):164-170, 125.

4. Silva PS, Reis ME, Aguiar VE, Fonseca MC. Unplanned extubation in the neonatal ICU: a systematic review, critical appraisal, and evidence-based recommendations. Respir Care 2013;58(7):12371245.

5. Merkel L, Beers K, Lewis MM, Stauffer J, Mujsce DJ, Kresch MJ. Reducing unplanned extubations in the NICU. Pediatrics 2014;133(5): e1367-e1372.

6. Costa AC, Schettino Rde C, Ferreira SC. Predictors of extubation failure and reintubation in newborn infants subjected to mechanical ventilation. Rev Bras Ter Intensiva 2014;26(1):51-56. 